\title{
Updated Information on the Local Group
}

\author{
Sidney van den Bergh \\ Dominion Astrophysical Observatory \\ National Research Council of Canada \\ 5071 West Saanich Road \\ Victoria, British Columbia, V8X 4M6 \\ Canada \\ e-mail: Sidney.vandenBergh@hia.nrc.ca
}

\begin{abstract}
The present note updates the information published in my recent monograph on The Galaxies of the Local Group. Highlights include (1) the addition of the newly discovered Cetus dwarf spheroidal as a certain member of the Local Group, (2) an improved distance for SagDIG, which now places this object very close to the edge of the Local Group zero-velocity surface, (3) more information on the evolutionary histories of some individual Local Group members, and (4) improved distance determinations to, and luminosities for, a number of Local Group members. These data increase the number of certain (or probable) Local Group members to 36. The spatial distribution of these galaxies supports Hubble's claim that the Local Group "is isolated in the general field." Presently available evidence suggests that star formation continued much longer in many dwarf spheroidals than it did in the main body of the Galactic halo. It is suggested that "young" globular clusters, such as Ruprecht 106, might have formed in now defunct dwarf spheroidals. Assuming SagDIG, which is the most remote Local Group galaxy, to lie on, or just inside, the zero-velocity surface of the Local Group yields a dynamical age $\gtrsim 17.9 \pm 2.7$ Gyr.
\end{abstract}

Subject headings: galaxies: clusters: general — Local Group

\section{Introduction}

The study of individual nearby galaxies that belong to the Local Group is presently

one of the most active areas of extragalactic research. As a result the recent reviews by van den Bergh (1999a, 2000) are already slightly out of date. The purpose of the present paper is to provide an updated report on the status of the most recent research on individual Local Group members, and on the structure of the Local Group itself. 


\section{Information on Individual Galaxies}

The individual galaxies for which new information has become available are listed below in approximate order of decreasing luminosity.

\subsection{The Andromeda galaxy (M31 = NGC 224)}

Kormendy \& Bender (1999) have used spectroscopy with high angular resolution to study the binary nucleus of M31. Their observations support Tremaine's model in which $\mathrm{P}_{1}$ is the brightest part of a single eccentric disk, where stars linger while at the apocenters of their orbits around $\mathrm{P}_{2}$. The latter object is found to contain a $3.3 \times 10^{7} \mathrm{M}_{\odot}$ black hole. Barmby et al. (1999) have published a catalog of 435 probable globular clusters in M31, of which 330 have UBV photometry, and 158 have been observed spectroscopically. These authors find that the metal-poor clusters have larger projected distances from the galaxy center, and show lower rotation, than do the metal-rich clusters. They estimate that M31 contains $450 \pm 100$ globulars, from which the specific globular cluster frequency $\mathrm{S}=0.9 \pm 0.2$. Hamilton \& Fesen (1999) have used the Hubble Space Telescope to image absorption by the remnant of the supernova S Andromedae (SN 1885A), which is observed to have a diameter of $0^{\prime \prime} .55 \pm 0^{\prime \prime} .15$, in the light of Fe II. From their observations these authors conclude that this remnant contains between 0.1 and $1.0 \mathrm{M}_{\odot}$ of iron.

\subsection{The Milky Way system}

Many years ago Eggen \& Sandage (1959) suggested that RR Lyrae and Groombridge 1830 (HR 4550) belong to a small physical group of five stars that are traversing the solar neighborhood at high velocity. The view that such clumps of high-velocity stars are real is supported by observations with the HIPPARCOS satellite (Helmi et al. 1999). From space motions of a nearly complete sample of nearby high-velocity halo stars these authors conclude that seven objects are members of a single debris stream. From their data Helmi et al. conclude that $\sim 8 \%$ of all metal-poor stars outside the solar radius represent the remnants of a single disintegrated dwarf galaxy. However, this estimate may turn out to be too high if a single narrow debris stream presently happens to be passing through the solar neighborhood.

Figer et al. (1999) have determined the slope of the mass spectrum of in the Arches and Quintuplet clusters, which are located near the Galactic center. They find that the mass spectra of these very young clusters have a slope $\Gamma=-0.65$, which is less steep than 
that for young clusters elsewhere in the Galaxy which typically have $\Gamma \sim-1.4$. Each cluster has a mass of $\sim 1 \times 10^{4} \mathrm{M}_{\odot}$, which are among the highest known in the Galaxy. Taken at face value these results suggest that the region near the Galactic center is particularly prone to forming very massive open clusters. From near-infrared echelle spectra Carr, Sellgren $\&$ Balachandran (1999) find that $[\mathrm{Fe} / \mathrm{H}]=-0.02 \pm 0.13$, i.e. nearly solar, for the Galactic center supergiant IRS 7.

\subsection{The Triangulum galaxy (M33 = NGC 598)}

Corbelli \& Salucci (1999) have measured the rotation curve of M33 out to a distance of $16 \mathrm{kpc}$ (13 disk scale-lengths) and find that the rotation curve rises out to the last measured point. This result implies a dark halo mass of $\gtrsim 5 \times 10^{10} \mathrm{M}_{\odot}$. Beyond $3 \mathrm{kpc}$ the gravitational potential is dominated by a dark halo with a density that decreases radially as $\mathrm{R}^{-1.3}$. From an unbiased sample of 60 clusters Chandar et al. (1999a,b) find that cluster formation in M 33 has been continuous over the last 10 Gyr, i.e. unlike the LMC, M 33 did not have a gap in its cluster formation history. Young clusters in M 33 have masses in the range $6 \times 10^{2} \mathrm{M}_{\odot}$ to $2 \times 10^{4} \mathrm{M}_{\odot}$, which is smaller than those of the old clusters which typically have masses of a few $\times 10^{5} \mathrm{M}_{\odot}$. Gordon et al. (1999) have doubled the sample of supernova remnants that are known in the Triangulum galaxy. Among their sample of 53 SNRs they find no evidence for a strong correlation between surface brightness and diameter. Many of these remnants are found to be associated with, or embedded in, H II regions. This (not unexpectedly) suggests that the majority of these remnants were produced by supernovae of Type II.

\subsection{The Large Magellanic Cloud (LMC)}

Detached eclipsing variables are powerful tools for the determination of extragalactic distances. Recently Nelson et al. (1999) have redetermined the reddening of the eclipsing variable HV 2274 and find $\mathrm{E}(\mathrm{B}-\mathrm{V})=0.083 \pm 0.006$, which is significantly lower than previously published values. From their new reddening Nelson et al. derive a distance modulus $(\mathrm{m}-\mathrm{M})_{0}=18.40 \pm 0.07$. Gibson (1999) has reviewed recent distance determinations

for the LMC, which range from $(\mathrm{m}-\mathrm{M})_{0}=18.20$ to 18.75 . This large spread shows that significant unappreciated sources of systematic error still exist in modern determinations of the distance to the Clouds of Magellan.

Sakai, Zaritsky \& Kennicutt (1999) have recently used the magnitude level of the tip of 
the LMC red giant branch to derive a distance modulus $(\mathrm{m}-\mathrm{M})_{0}=18.59 \pm 0.09$ (random) \pm 0.16 (systematic), which agrees well with previous determinations via Cepheid variables.

Dolphin (1999b) has studied the star formation history in two fields in the LMC. His results, which are summarized in Table 1, appear to show (1) a steady rise of the metallicity index $[\mathrm{Fe} / \mathrm{H}]$ with time, and (2) that the rate of star formation between 2.5 and 7 Gyr ago was an order of magnitude lower than it has been during the most recent 2-3 Gyr period. Since the two fields studied by Dolphin are separated by $3^{\circ} .0(2.6 \mathrm{kpc})$ his data refer to global star formation rates. Taken at face value Dolphin's results appear to weaken the previous conclusion that the rate of cluster formation in the Large Cloud increased more rapidly $\sim 3$ Gyr ago than did the rate of star formation. However, Holtzman et al. (1999) reach a very different conclusion from Hubble Space Telescope observations of two fields in the outer disk of the LMC. They conclude that there was no gap in the age distribution of these stars. Clearly we are still far from understanding the evolutionary history of the disk component of the LMC. From new HST color- magnitude diagrams Johnson et al. (1999) find that the age difference between the Large Cloud clusters NGC 1466, NGC 2257 and Hodge 11, on the one hand, and the Galactic clusters M 92 and M 3 on the other, is less than $1.5 \mathrm{Gyr}$. It is presently not understood why globular cluster formation occurred simultaneously in the LMC, the main body of the Galactic halo, and in the outer Galactic halo (NGC 2419).

From the relatively large number of red clump stars in the LMC Bar Holtzman et al. conclude that the stellar population in the Bar is older than that in the outer fields of the Large Cloud. This conflicts with some previous work that had suggested that the LMC Bar might be a relatively recent feature. From their integrated spectra Dutra et al. (1999) conclude that NGC $1928([\mathrm{Fe} / \mathrm{H}]=-1.2)$ and NGC $1939([\mathrm{Fe} / \mathrm{H}]=-2.0)$ may be globular clusters that had not previously been recognized as such. If confirmed this would increase the number of LMC globular clusters from 13 to 15. A catalog of X-ray sources in the LMC has been published by Haberl \& Pietsch (1999). These authors give likely identifications for 144 sources. Of these objects 46 (32\%) appear to be associated with supernova remnants, $17(12 \%)$ are X-ray binaries, and nine $(6 \%)$ are "supersoft" sources. The majority of unidentified sources are probably associated with background galaxies or foreground stars. Demers \& Battinelli (1999) have surveyed the periphery of the LMC for young blue stars that might be associated with the Bridge linking the Large and the Small Clouds. Few such stars are found suggesting that the Bridge does not extend deep into the LMC. Liebert (1999) has pointed out that the hot blue star found in the LMC cluster NGC 1818 has the wrong luminosity and radius to be a luminous white dwarf.

Murali (1999) finds that the motion of the Magellanic Stream through ambient gas can 
strongly heat the Stream clouds, driving mass loss and causing evaporation. Survival of the stream for 500 Myr sets an upper limit $<10^{-5} \mathrm{~cm}^{-3}$ for the Galactic halo gas through which the Stream in orbiting.

\subsection{The Small Magellanic Cloud}

Most previous investigators have concluded that cluster formation in the Small Cloud has proceeded at a more-or-less uniform rate. However new cluster age determinations by Rich et al. (1999) now suggest that cluster formation in the SMC may have been enhanced during bursts that occurred 2 Gyr and 8 Gyr ago. More, and more accurate, age determinations for Small Cloud clusters will be required to strengthen this conclusion. An unbiased survey of 93 star clusters in a 2.4 square degree area of the SMC (Pieterzyński \& Udalski 1999) shows an age distribution that is very strongly biased towards young clusters, with only $3 \%$ of the clusters having ages $>1$ Gyr. The fact that $60 \%$ of all SMC clusters are younger than $100 \mathrm{Myr}$ should probably be interpreted as evidence for short cluster life-times, rather than as evidence for a recent burst of cluster formation.

Bica \& Dutra (1999) have published an updated census of SMC clusters, and of clusters in the Bridge between the LMC and SMC, that is based on the OGLE survey. Their paper contains a map of the distribution of SMC clusters, which shows a strong concentration in the Small Cloud "Bar" and a lesser concentration of clusters in the Wing of the SMC.

Cold atomic hydrogen has been detected in the Bridge between the Magellanic Clouds by Kobulnicky \& Dickey (1999). The early-type stars observed in the Bridge could have formed in these cold clouds. These objects therefore need not have migrated from the main body of the SMC.

Rolleston et al. (1999) have compared the abundances of three early-type stars in the Bridge between the LMC and the SMC with those observed in "normal" B-type stars near the Sun. They found an average metal deficiency $[\mathrm{m} / \mathrm{H}]=-1.05 \pm 0.12$ for these objects. Surprisingly this value falls well below the present (Luck et al. 1998) SMC metallicity $[\mathrm{Fe} / \mathrm{H}]=-0.74$. In fact it lies between that of the globular cluster NGC $121([\mathrm{Fe} / \mathrm{H}]=-1.19)$, which has an age of $\sim 12 \mathrm{Gyr}$, and that of the old open cluster L $1([\mathrm{Fe} / \mathrm{H}]=-1.01)$ that has an age of $\sim 10 \mathrm{Gyr}$. Taken at face value this result suggests that the material in the Bridge was tidally detached from the Small Cloud 10 Gyr ago. However, Putman (1999) has argued that the Bridge was formed only 0.2 Gyr ago, when the LMC and SMC had a very close encounter with a minimum separation of only $\sim 7 \mathrm{kpc}$. Alternatively it might be hypothesized that the metallicity 
of gas in the Bridge was lowered by swept-up pristine inter-galactic gas. The metallicity of NGC 330, which is the most luminous young SMC cluster, remains controversial. Hill (1999) finds $\langle[\mathrm{Fe} / \mathrm{H}]\rangle=-0.82 \pm 0.11$ for six cool cluster supergiants, compared to $\langle[\mathrm{Fe} / \mathrm{H}]\rangle=-0.69 \pm 0.10$ for six cool SMC field stars. Finally Gonzalez \& Wallerstein (1999) obtain $\langle[\mathrm{Fe} / \mathrm{H}]\rangle=-0.94 \pm 0.02$ for seven stars in NGC 330 .

The ninth nova to be discovered in the SMC was found near the optical center of this galaxy by Glicenstein (1999) at $\alpha=0^{\mathrm{h}} 59^{\mathrm{m}} 23 .{ }^{\mathrm{s}} 0, \delta=-73^{\circ} 07^{\prime} 56^{\prime \prime}$ (equinox 2000).

\subsection{The spheroidal' galaxy NGC 205}

A nova has been discovered in NGC 205 by Johnson \& Modjaz (1999).

\subsection{The starburst galaxy IC 10}

From observations in the far infrared Bolatto et al. (1999) find that dust in the mild starburst galaxy IC 10 appears to deficient in small grains. It seems likely that such grains were destroyed by intense UV radiation in the neighborhood of the hot luminous stars that were formed during the recent burst of star formation in this galaxy.

\subsection{The spheroidal galaxy NGC 185}

The history of star formation in NGC 185 has been studied by Martínez-Delgado, Aparicio \& Gallart (1999), who find that the bulk of the star formation in this galaxy took place at early times. Stars only formed near the center of this galaxy during the last $\sim 1$ Gyr. Most of the young blue objects discovered by Baade (1951) turn out to be star clusters, rather than individual stars. Martínez-Delgado et al. have also discovered a supernova remnant near the center of NGC 185.

\footnotetext{
${ }^{1}$ The galaxies NGC 147, NGC 185, and NGC 205 belong to the same morphological family as do the dwarf spheroidals, such as Sculptor and Fornax. However, their relatively high luminosity makes the term dwarf spheroidal seem inappropriate. I therefore call such objects "spheroidals". It seems likely that such spheroidals are lower luminosity examples of the form family that de Vaucouleurs (1959) refers to as "lenticulars".
} 


\subsection{The dwarf irregular IC 1613}

Hubble Space Telescope observations by Cole et al. (1999) show that the dominant stellar population in IC 1613 has an age of $\sim 7$ Gyr. From its Hess diagram these authors find that the evolutionary history of IC 1613 may have been similar to that of the Pegasus dwarf (DDO 216). Both of these objects are classified Ir V on the DDO system. Antonello et al. (1999) have found five Cepheids of Population II in IC 1613, thus providing prima facie evidence for the existence of a very old stellar population component in this galaxy. King, Modjaz \& Li (1999) have observed what is believed to be the first nova ever observed in IC 1613.

\subsection{The Wolf-Lundmark-Melotte system (DDO 221)}

A recent color-magnitude diagram obtained with the Hubble Space Telescope (Dolphin 1999a) shows that about half of all star formation in the WLM galaxy occurred during a burst that began $\sim 13$ Gyr ago. During the course of this burst the metallicity increased from $[\mathrm{Fe} / \mathrm{H}] \sim-2.2$ to $[\mathrm{Fe} / \mathrm{H}]=-1.3$. From the apparent absence of a horizontal branch population Dolphin places an upper limit of $\sim 20 \mathrm{M}_{\odot}$ per Myr on the rate of star formation between 12 Gyr and 15 Gyr ago. Between 2.5 and 9 Gyr ago the average rate of star formation was $100-200 \mathrm{M}_{\odot}$ per Myr.

The WLM system contains a single globular cluster, for which Hodge et al. (1999) find $\mathrm{M}_{\mathrm{V}}=-8.8,[\mathrm{Fe} / \mathrm{H}]=-1.5$ and an age of $\sim 15$ Gyr. It is of interest to note that the lone globular in this dwarf galaxy has a luminosity that falls slightly above that of the mean for all globular clusters. The apparent absence of faint low-mass globulars is of particular interest because destruction of such objects probably can not be attributed to the weak tidal forces of the WLM dwarf galaxy.

\subsection{The disintegrating Sagittarius galaxy}

From observations of tidal debris of the Sagittarius system Johnston et al. (1999) conclude that this object has orbited the Galaxy for at least 1 Gyr, and that the mass of this galaxy has decreased by a factor of 2-3 during this period. The orbits of Sgr are found to have Galactocentric distances that oscillate between $\sim 13 \mathrm{kpc}$ and $\sim 41 \mathrm{kpc}$ and periods in the range 550-750 Myr. Its most recent perigalacticon occurred $50 \mathrm{Myr}$ ago. [Jiang \& Binney (1999) find that the Sagittarius dwarf might have started its infall from a distance greater than $200 \mathrm{kpc}$ if its initial mass was as great as $\left.\sim 10^{11} \mathrm{M}_{\odot}.\right]$ Both the orbit 
of Sgr, and the Galactic potential field, could be constrained by improved proper motion observations of the stellar debris associated with this object. Burton \& Lockman (1999) have found no neutral hydrogen gas associated with the Sagittarius dwarf.

\subsection{The Fornax dwarf spheroidal}

Buonanno et al. (1999) have recently used the Hubble Space Telescope to obtain a color-magnitude diagram for the globular cluster Fornax No. 4. Whereas the clusters

Fornax 1, 2, 3 and 5 have horizontal branches that extend over a wide range of colors (and include RR Lyrae variables), Fornax 4 is seen to have a red horizontal branch. Fornax 4 is 3 Gyr younger than the other Fornax globulars. Buonanno et al. draw attention to the fact that the color-magnitude diagram of Fornax 4 exhibits a strong resemblance to that of the "young" Galactic globular cluster Ruprecht 106 (Fusi Pecci et al. 1995, and references therein). This observation raises two questions: (1) What is it about dwarf spheroidals that allows them to form globular clusters such as Fornax 4 and Terzan 7 (which is associated with the Sagittarius dwarf) long after the formation of globulars ceased in the main body of the Galactic halo, and (2) could it be that "young" outer halo globulars such as Ruprecht 106 were originally formed in now defunct dwarf spheroidals?

\subsection{The Sagittarius dwarf irregular galaxy (SagDIG)}

On the basis of a very uncertain distance of 1.4 Mpc SagDIG (UKS 1927-177= UGA 438) had previously been regarded as a possible Local Group member. New photometry by Lee \& Kim (1999) yields a distance (based on the tip of the red giant branch) of only $1.18 \pm 0.10 \mathrm{Mpc}$, and an improved integrated luminosity $\mathrm{M}_{\mathrm{V}}=-11.97$, which makes this object slightly more luminous that Leo A $\left(\mathrm{M}_{\mathrm{V}}=-11.5\right)$. A similar distance of $1.06 \pm 0.10$ Mpc has recently been found by Karachentsev, Aparicio \& Makarova (1999). If, following Courteau \& van den Bergh (1999), we assume that the barycenter of the Local Group is situated at a distance of $454 \mathrm{kpc}$ towards $\ell=121 .^{\circ} 7, \mathrm{~b}=-21 .^{\circ} 3$, then SagDIG is located at a distance of $1.29 \pm 0.09 \mathrm{Mpc}$ from the LG barycenter. This makes SagDIG the most remote object suspected of Local Group membership. Its distance is marginally greater than the radius of the zero-velocity surface of the Local Group, for which Courteau \& van den Bergh (1999) find a value of $\mathrm{R}_{0}=1.18 \pm 0.15 \mathrm{Mpc}$. 


\subsection{The Leo I (Regulus system)}

From HST observations Gallart et al. (1999) conclude that 70\% - 80\% of the star forming activity in Leo I took place between $7 \mathrm{Gyr}$ and $1 \mathrm{Gyr}$ ago. There is little or no evidence for the presence of stars with ages $>10$ Gyr. About 1 Gyr ago the rate of star formation appears to have dropped abruptly to a near-negligible level. However, some very low-level star formation may have continued until 300 Myr ago.

\subsection{The M31 companion And II}

From spectra of seven stars in And II, that were obtained with the Keck telescope, Côté et al. (1999) have found a mean velocity $\mathrm{V}_{\mathrm{r}}=-188 \pm 3 \mathrm{~km} \mathrm{~s}^{-1}$, and a velocity dispersion of $9.3 \pm 2.6 \mathrm{~km} \mathrm{~s}^{-1}$. From these data they obtain a mass-to-light ratio $\mathrm{M} / \mathrm{L}_{\mathrm{V}}=21_{-10}^{+14}$ in solar units, i.e. this dwarf spheroidal appears to contain a significant amount of dark matter. Côté, Oke \& Cohen (1999) have also obtained Keck spectra of 42 red giants in And II, from which they find a mean metallicity $\langle[\mathrm{Fe} / \mathrm{H}]\rangle=-1.47 \pm 0.19$, with a dispersion of $0.35 \pm 0.10$ dex. Da Costa et al. (1999) have studied the color-magnitude diagram of And II and find that the majority of stars have ages in the range 6 Gyr to 9 Gyr, although the presence of RR Lyrae variables and blue horizontal branch stars attests to the existence of a population component with an age $>10$ Gyr. And II differs from And I in that it does not exhibit a radial gradient in horizontal branch morphology. Furthermore the dispersion in abundance is considerably larger in And II than it was found to be in And I. These results show that these two dwarf spheroidal companions to M 31 must have had quite different evolutionary histories. It would be interesting to know if there is a correlation between a radial horizontal branch gradient and the metallicity dispersion among dwarf

spheroidal galaxies. On the basis of its $680 \mathrm{kpc}$ distance Da Costa et al. conclude that And II is physically associated with M 31, rather than with M 33.

\subsection{The dwarf spheroidals And V, And VI and And VII}

Caldwell (1999) has derived accurate luminosities and surface brightness profiles for Andromeda V, VI and VII. And V turns out to be fainter than previously believed, whereas And VI and And VII were found to be more luminous than previously thought. And V has a metallicity that lies above the average metallicity versus luminosity relation for Local Group dwarf galaxies. 


\subsection{The Aquarius dwarf (AqrDIG = DDO 210)}

The membership of this dwarf irregular in the Local Group has now been firmly established by Lee et al. (1999), who derive a distance of $950 \pm 50 \mathrm{kpc}$ from the magnitude of the giants at the tip of the red giant branch. The corresponding distance of this object from the barycenter of the Local Group is also $950 \mathrm{kpc}$. This implies that DDO 210 is rather isolated in space.

\subsection{The recently discovered Cetus system}

Whiting, Hau \& Irwin (1999) have searched the region with $\delta<+3^{\circ}$ for faint dwarfs that might be members of the Local Group. Two objects were discovered during this program. One of these was the Antlia system, which lies just beyond the zero-velocity surface of the Local Group (van den Bergh 1999b). The other was a faint dwarf spheroidal galaxy in Cetus. From the position of the tip of the giant branch of the Cetus system Whiting et al. derive a Galactocentric distance of $775 \pm 50 \mathrm{kpc}$, and a distance of $615 \mathrm{kpc}$ from the adopted center of the Local Group. This places Cetus comfortably inside the 1.18 Mpc radius (Courteau \& van den Bergh 1999) of the Local Group zero-velocity surface. It would be of great importance to obtain radial velocities for individual red giants in the Cetus dwarf. This would enable one to determine the amount of dark matter in this galaxy. Furthermore knowledge of the systemic velocity of Cetus would add weight to the Local Group mass determination. No neutral hydrogen gas has been found to be associated with the Cetus dwarf.

\subsection{The Sculptor dwarf spheroidal}

Majewski et al. (1999) find that the metallicity distribution in Sculptor appears to be bimodal with components having $[\mathrm{Fe} / \mathrm{H}]=-2.3$ and $[\mathrm{Fe} / \mathrm{H}]=-1.5$. As is the case in many other Local Group galaxies the older metal-poor component appears more extended than the younger metal-rich component. However, Hurley-Keller, Mateo \& Grebel (1999),

although confirming the central concentration of red horizontal branch stars, do not find a radial age (or metallicity) gradient. 


\subsection{The Phoenix dwarf galaxy}

Martínez-Delgado, Gallart \& Aparicio (1999) find that this dIr/dSph galaxy has an inner component that contains young stars which is stretched in the east-west direction, and an outer component that is extended north-south, which is mainly populated by old stars. The rate of star formation in the central region of this galaxy appears to have remained approximately constant over time. St.-Germain et al. (1999) have found a cloud of $\sim 10^{5} \mathrm{M}_{\odot}$ of $\mathrm{H}$ I located just west of Phoenix, that has a radial velocity of $-23 \mathrm{~km} \mathrm{~s}^{-1}$, which may be physically associated with this object. Optical radial velocities of stars in Phoenix will be required to confirm this suspicion.

\subsection{The Ursa Minor dwarf}

New Hubble Space Telescope observations by Mighell \& Burke (1999) confirm that the UMi system had a simple evolutionary history with a single $\sim 2$ Gyr long burst of star formation occurring 14 Gyr ago.

\section{DISCUSSION}

New data by Armandroff et al. (1999), Caldwell (1999), Da Costa et al. (1999), Dolphin (1999a), Lee \& Kim (1990), Lee et al. (1999), and Whiting et al. (1999) are incorporated in Table 2, which lists classification types on the DDO system, luminosities, Galactocentric distances, and distances from the adopted barycenter, for all 36 presently known Local Group members. Figure 1 shows a plot of the integral frequency of galaxy distances from the Local Group barycenter adopted by Courteau \& van den Bergh (1999). To minimize the influence of observational selection effects galaxies with $\mathrm{M}_{\mathrm{V}}>-10.0$ have not been plotted. The figure shows that only one galaxy (SagDIG at $\mathrm{R}_{\mathrm{LG}}=1.29 \pm 0.09 \mathrm{Mpc}$ ) lies marginally beyond the dynamically determined radius of the Local Group zero-velocity surface, for which Courteau \& van den Bergh (1999) determined $R_{0}=1.10 \pm 0.15$ Mpc. From Eqn. (10) of Courteau \& van den Bergh (1999) it is seen that the age of the Local Group can be determined from its mass $\mathrm{M}$, and the radius of its zero-velocity surface. Adopting a Local Group mass $\mathrm{M}=(2.3 \pm 0.6) \times 10^{12} \mathrm{M}_{\odot}$ and assuming that SagDIG, which is located at a distance of $1.29 \pm 0.09 \mathrm{Mpc}$ from the barycenter of the Local Group, lies on the Local Group zero-velocity surface one obtains a value of $17.9 \pm 2.7 \mathrm{Gyr}$ for the dynamical age of the Local Group. This value compares favorably with the 12-16 Gyr age derived from evolutionary models of the most metal-poor stars, the $16 \pm 6$ Gyr age that Sneden et al. (1996) derive 
from the Th/Eu abundance ratio in CS 22893-052, and the 10-20 Gyr ages that Cowan, Thielemann \& Truran (1991) have derived from cosmochronology. If the Local Group zero-velocity surface, in fact, lies slightly beyond SagDIG, then the LG age calculated above becomes a lower limit. The figure shows that $71 \%$ of all Local Group galaxies are situated within $0.5 \mathrm{Mpc}$ of the Local Group barycenter. On the other hand only one (4\%) of the known Local Group galaxies brighter than $\mathrm{M}_{\mathrm{V}}=-10$ are located beyond $\mathrm{R}_{\mathrm{LG}}=1.0 \mathrm{Mpc}$. The total luminosity of the Local Group is found to be $\mathrm{M}_{\mathrm{V}}=-22.0$, of which only $0.5 \%$ originates beyond $\mathrm{R}_{\mathrm{LG}}=0.5 \mathrm{Mpc}$. This strongly supports Hubble's (1936) claim that the Local Group "is isolated in the general field". The only Local Group member, in addition to SagDIG, that is known to have $R_{\mathrm{LG}}>1.0 \mathrm{Mpc}$ is the faint Tucanae system $\left(\mathrm{M}_{\mathrm{V}}=-9.6\right)$ at $\mathrm{R}_{\mathrm{LG}}=1.1 \mathrm{Mpc}$.

Among the most important Local Group problems that remain to be resolved are the following:

- Why does the luminosity distribution of Local Group galaxies contain at least an order of magnitude fewer faint dwarfs than theory (Klypin et al. 1999) predicts?

Is theory wrong, or have the majority of low-mass dwarfs formed no stars? A deep search for stars associated with compact high-velocity clouds might answer this question.

- Why does the Large Magellanic Cloud contain very old globular clusters that have disk kinematics, while M 33 is embedded in an apparently younger globular cluster system that exhibits halo kinematics?

- Why did the LMC form so few clusters with ages between 4 Gyr and 10 Gyr? Was there a similar hiatus in the rate of star formation, or did the fraction of all Large Cloud stars that ended up in clusters suddenly increase $\sim 4$ Gyr ago?

- Did any of the Local Group galaxies change their morphological type during the last $\sim 10$ Gyr? It would be particularly important to establish if the Bar of the LMC is a relatively young feature.

- Why did different Local Group dwarf galaxies have such different star forming histories, and how do such differences depend on environment?

\footnotetext{
${ }^{2}$ In van den Bergh (2000) all galaxies within $~ 1.5 \mathrm{Mpc}$ were initially regarded as candidate Local Group members. Subsequently it was found that the Local Group has a zero-velocity surface with a radius of $1.10 \pm 0.15 \mathrm{Mpc}$, as measured from its barycenter. All galaxies within 1.25 Mpc of the barycenter have therefore been regarded as possible, or probable, Local Group members.
} 
- It would be important to strengthen and confirm Freeman's (1999) conclusion that the metal-rich $\mathrm{r}^{1 / 4}$ halo of $\mathrm{M} 31$ resulted from violent relaxation after the merger of two massive ancestral galaxies, whereas the metal-poor outer halo of the Milky Way system was mainly built up via capture of numerous low-mass metal-poor objects.

The new generation of powerful optical, radio and space telescopes, in conjunction with improved wide-field and infrared-sensitive detectors, should enable us to answer many of these questions in the first years of the next millennium.

I am indebted to Alan Whiting for making his data on the Cetus system available before publication. 


\section{REFERENCES}

Antonello, E., Mantegazza, L., Fugazza, D., Bossi, M., \& Covino, S. 1999, A\&A,349, 55

Armandroff, T.E., Jacoby, G.H. \& Davies, J.E. 1999, AJ, 118, 1220

Baade, W. 1951, Publ. Univ. Michigan, Vol. X, p. 7

Barmby, P., Huchra, J.P., Brodie, J.P., Forbes, D.A., Schroeder, L.L. \& Grillmair, C.J. 1999, astro-ph/9911152

Bica, E. \& Dutra, C.M. 1999, astro-ph/9912386

Bolatto, A.D., Jackson, J.M., Wilson, C.D. \& Moriarty-Schieven, G. 1999, astro-ph/9910564

Buonanno, R., Corsi, C.E., Castellani, M., Marconi, G., Fusi Pecci, F. \& Zinn, R. 1999, AJ, 118,1671

Burton, W.B. \& Lockman, F.J. 1999, A\&A, 349, 7

Caldwell, N. 1999, AJ, 118, 1230

Carr, J.S., Sellgren, K. \& Balachandrian, B.C. 1999, astro-ph/9909037

Chandar, R., Bianchi, L. \& Ford, H.C. 1999a, ApJ, 517, 668

Chandar, R., Bianchi, L., Ford, H.C. \& Salasnich, B. 1999b, PASP, 111, 794

Cole, A.A. et al. 1999, AJ, 118, 1657

Corbelli, E. \& Salucci, P. 1999, astro-ph/9909252

Côté, P., Mateo, M., Olszewski, E.W. \& Cook, K.H. 1999, ApJ, 526, 147

Côté, P., Oke, J.B. \& Cohen, J.G. 1999, AJ, 118, 1645

Courteau, S. \& van den Bergh, S. 1999, AJ, 118, 345

Cowan, J.J., Thielemann, F.-K. \& Truran, J.W. 1991, ARAA, 29, 447

Da Costa, G.S., Armandroff, T.E., Caldwell, B. \& Seitzer, P. 1999, astro-ph/9911020

Demers, S. \& Battinelli, P. 1999, AJ, 118, 1700

de Vaucouleurs, G. 1959, Handb. d. Astrophysik, 53, 311

Dolphin, A.E. 1999a, astro-ph/9910458

Dolphin, A.E. 1999b, astro-ph/9910524

Dutra, C.M., Bica, E., Clari(, J.J. \& Piatti, A.E. 1999, MNRAS, 305, 373

Eggen, O.J. \& Sandage, A.R. 1959, MNRAS, 119, 255

Figer, D.F., Kim, S.S., Morris, M., Serabyn, E., Rich, R.M. \& McLean, I.S. 1999, ApJ, 525, 750 
Freeman, K.C. 1999, in The Galactic Halo, eds. B.K. Gibson, T.S. Axelrod and M.E. Putman, (San Francisco: ASP), p. 167

Fusi Pecci, F., Bellazzinni, M., Cacciari, C. \& Ferraro, F.R. 1995, AJ, 110, 1664

Gallart, C., Freedman, W.L., Aparicio, A., Bertelli, G. \& Chiosi, C.1999, astro-ph/9906121

Gibson, B.K. 1999, astro-ph/9910574

Glicenstein, J.-F. 1999, IAU Circular No. 7286

Gonzalez, G. \& Wallerstein, G. 1999, AJ, 117, 2286

Gordon, S.M., Duric, N., Kirshner, R.P., Goss, W.M. \& Viallefond, F. 1999, ApJS, 120, 247

Haberl, F. \& Pietsch, W. 1999, A\&AS, 139, 277

Hamilton, A.J.S. \& Fesen, R.A. 1999, astro-ph/9907102

Helmi, A., White, S.D.M., de Zeeuw, P.T. \& Zhao, Z. 1999, Nature, 402, 29

Hill, V. 1999, A\&A, 345, 430

Hodge, P.W., Dolphin, A.E., Smith, T.R. \& Mateo, M. 1999, ApJ, 521, 577

Holtzman, J.A. et al. 1999, astro-ph/9907257

Hubble, E. 1936, The Realm of the Nebulae, (New Haven: Yale University Press), p. 125

Hurley-Keller, D., Mateo, M. \& Grebel, E. 1999, ApJ, 523, L25

Jiang, I.-G. \& Binney, J. 1999, astro-ph/9908025

Johnson, J.A., Bolte, M., Stetson, P.B., Hesser, J.E. \& Somerville, R.S. 1999, astroph/9911187

Johnson, R. \& Modjaz, M. 1999, IAU Circular No. 7240

Johnston, K.V., Majewski, S.R., Siegel, M.H., Reid, I.N. \& Kunkel, W.E. 1999, AJ, 118, 1717

Karachentsev, I., Aparicio, A. \& Markova, L. 1999, astro-ph/9910136

King, J.Y., Modjaz, M. \& Li, W.D. 1999, IAU Circular No. 7287

Klypin, A.A., Kravtsov, A.V., Venezuela, O. \& Prada, F. 1999, astro-ph/9901240

Kobulnicky, H.A. \& Dickey, J.M. 1999, AJ, 117, 908

Kormendy, J. \& Bender, R. 1999, ApJ, 522, 772

Lee, M.G., Aparicio, A., Tikonov, N., Byun, Y.-I. \& Kim, E. 1999, AJ, 118, 853

Lee, M.G. \& Kim, S.C. 1999, astro-ph/9910481

Liebert, J. 1999, ApJ, 514, L25 
Luck, R.E., Moffet, T.J., Barnes, T.G. \& Gieren, W.P. 1998, AJ, 115, 605

Majewski, S.R., Siegel, M.H., Patterson, R.J. \& Rood, R.T. 1999, ApJ, 520, L33

Martínez-Delgado, D., Gallart, C. \& Aparicio, A. 1999, AJ, 118, 862

Mighell, K.J. \& Burke, C.J. 1999, AJ, 118, 366

Murali, C. 1999, astro-ph/9912168

Nelson, C.A., Cook, K.H., Popowski, P. \& Alves, D.R. 1999, astro-ph/9910172

Pieterzy1/2ski, G. \& Udalski, A. 1999, astro-ph/9906339

Putman, M.E. 1999, astro-ph/99909080

Rich, R.M, Shara, M., Fall, S.M. \& Zurek, D. 1999, astro-ph/9907393

Rolleston, W.R.J., Dufton, P.L., McErlean, N.D. \& Venn, K.A. 1999, A\&A, 348, 728

Sakai, S., Zaritsky, D. \& Kennicutt, R.C. 1999, astro-ph/9911528

Sneden, C., McWilliam, A., Preston, G.W. \& Cowan, J.J. 1996, in Formation of the Galactic Halo (San Francisco: ASP), p. 387

St-Germain, J., Garignan, C., C(t(, S. \& Oosterlo, T. 1999, AJ, 118, 1235

van den Bergh, S. 1999a, A\&A Review, 9, 273

van den Bergh, S. 1999b, ApJ, 517, L97

van den Bergh, S. 2000, The Galaxies of the Local Group (Cambridge: Cambridge University Press)

Whiting, A.B., Hau, G. \& Irwin, M. 1999, AJ, 118, xxxx 
Table 1. STAR FORMATION HISTORY OF THE LARGE CLOUD ${ }^{a}$

\begin{tabular}{rrr}
\hline \hline Age $(\mathrm{Gyr})$ & $\mathrm{SFR}\left(\mathrm{M}_{\odot} / \mathrm{Myr}\right)$ & {$[\mathrm{Fe} / \mathrm{H}]^{\mathrm{b}}$} \\
\hline $0.0-1.0$ & $350 \pm 20$ & -0.38 \\
$1.0-2.5$ & $269 \pm 40$ & -0.54 \\
$2.5-5.0$ & $20 \pm 30$ & -0.83 \\
$5.0-7.0$ & $30 \pm 40$ & -1.06 \\
$7.0-9.0$ & $240 \pm 70$ & -1.32 \\
$9.0-12.0$ & $440 \pm 60$ & -1.63 \\
\hline
\end{tabular}

aData from Dolphin (1999b)

${ }^{\mathrm{b}}$ These metallicity values are uncertain by $\sim 0.1 \mathrm{dex}$ 
Table 2. DATA ON LOCAL GROUP GALAXIES ${ }^{a}$

\begin{tabular}{|c|c|c|c|c|c|}
\hline Name & Alias & Type & $\mathrm{M}_{\mathrm{V}}$ & D (Galaxy) & D (Local Group) \\
\hline WLM & DDO 221 & Ir IV-V & -14.4 & $0.95 \mathrm{Mpc}$ & $0.81 \mathrm{Mpc}$ \\
\hline IC 10 & UGC 192 & Ir IV: & -16.3 & 0.66 & 0.27 \\
\hline Cetus & & dSph & -10.1 & 0.78 & 0.62 \\
\hline NGC 147 & UGC 326 & Sph & -15.1 & 0.66 & 0.22 \\
\hline And III & $\mathrm{A} 0032+36$ & dSph & -10.2 & 0.76 & 0.31 \\
\hline NGC 185 & UGC 396 & $\mathrm{Sph}$ & -15.6 & 0.66 & 0.22 \\
\hline NGC 205 & M 110 & Sph & -16.4 & 0.76 & 0.31 \\
\hline M 32 & NGC 221 & $\mathrm{E} 2$ & -16.5 & 0.76 & 0.31 \\
\hline M 31 & NGC 224 & Sb I-II & -21.2 & 0.76 & 0.30 \\
\hline And I & $\mathrm{A} 0043+37$ & $\mathrm{dSph}$ & -11.8 & 0.81 & 0.36 \\
\hline $\mathrm{SMC}$ & & Ir IV/IV-V & -17.1 & 0.06 & 0.48 \\
\hline Sculptor & & $\mathrm{dSph}$ & -9.8 & 0.09 & 0.44 \\
\hline Pisces & LGS 3 & $\mathrm{dIr} / \mathrm{dSph}$ & -10.4 & 0.81 & 0.42 \\
\hline IC 1613 & & Ir V & -15.3 & 0.72 & 0.47 \\
\hline And $\mathrm{V}$ & & dSph & -9.1 & 0.81 & 0.37 \\
\hline And II & & $\mathrm{dSph}$ & -11.8 & 0.68 & 0.26 \\
\hline M 33 & NGC 598 & Sc II-III & -18.9 & 0.79 & 0.37 \\
\hline Phoenix & & $\mathrm{dIr} / \mathrm{dSph}$ & -9.8 & 0.40 & 0.59 \\
\hline Fornax & & $\mathrm{dSph}$ & -13.1 & 0.14 & 0.45 \\
\hline LMC & & Ir III-IV & -18.5 & 0.05 & 0.48 \\
\hline Carina & & $\mathrm{dSph}$ & -9.4 & 0.10 & 0.51 \\
\hline Leo A & DDO 69 & Ir $\mathrm{V}$ & -11.5 & 0.69 & 0.88 \\
\hline Leo I & Regulus & $\mathrm{dSph}$ & -11.9 & 0.25 & 0.61 \\
\hline Sextans & & $\mathrm{dSph}$ & -9.5 & 0.09 & 0.51 \\
\hline Leo II & DDO 93 & $\mathrm{dSph}$ & -10.1 & 0.21 & 0.57 \\
\hline Ursa Minor & DDO 199 & dSph & -8.9 & 0.06 & 0.43 \\
\hline Draco & DDO 208 & $\mathrm{dSph}$ & -8.6 & 0.08 & 0.43 \\
\hline Milky Way & Galaxy & $\mathrm{S}(\mathrm{B}) \mathrm{bc} \mathrm{I}-\mathrm{II}$ & $-20.9:$ & 0.01 & 0.46 \\
\hline Sagittarius & & $\mathrm{dSph}(\mathrm{t})$ & $-13.8::$ & 0.03 & 0.46 \\
\hline SagDIG & & Ir $\mathrm{V}$ & -12.0 & 1.18 & 1.29 \\
\hline NGC 6822 & & Ir IV-V & -16.0 & 0.50 & 0.67 \\
\hline Aquarius & DDO 210 & $\mathrm{~V}$ & -10.9 & 0.95 & 0.95 \\
\hline Tucanae & & dSph & -9.6 & 0.87 & 1.10 \\
\hline Cassiopeia & And VII & $\mathrm{dSph}$ & -12.0 & 0.69 & 0.29 \\
\hline Pegasus & DDO 216 & Ir $\mathrm{V}$ & -12.3 & 0.76 & 0.44 \\
\hline Pegasus II & And VI & $\mathrm{dSph}$ & -11.3 & 0.78 & 0.38 \\
\hline
\end{tabular}

${ }^{\mathrm{a}}$ Galaxies listed in order of increasing Right Ascension 


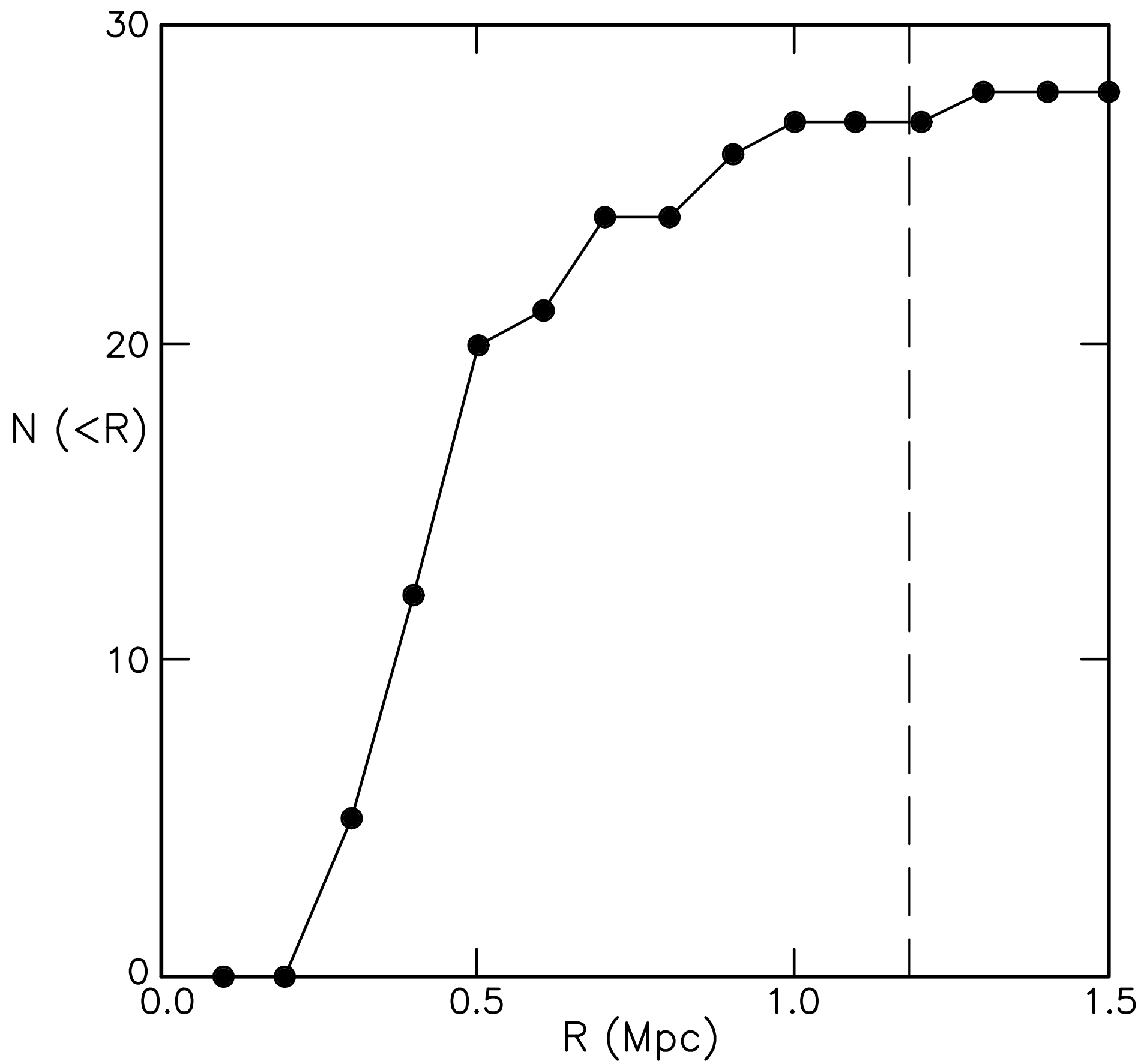

Fig. 1.- Number of Local Group members within a distance $\mathrm{R}$ of the barycenter of the Local Group. To minimize the effects of observational selection galaxies fainter than $\mathrm{M}_{\mathrm{V}}=-10.0$ were excluded. The dashed vertical line is the dynamically determined radius of the Local Group zero-velocity surface. 